원 저

\title{
肺結核患者의 換氣力學
}

\author{
慶北大學校 醫科大學 內科學敎室 \\ 尹 德 求・丁 泰 勳・朴 熙 明
}

$=$ Abstract $=$

\section{Ventilatory Dynamics in Pulmonary Tuberculosis}

Duk Koo Yun, M.D., Tae Hoon Jung, M.D. and Hi Myung Park, M.D.

Department of Internal Medicine, School of Medicine, Kyungpook National University

Taegu, Korea

\begin{abstract}
Ventilatory dynamics was studied in patients with pulmonary tuberculosis by means of analyses of simultaneously recorded forced expiratory volume (FEV) and maximal expiratory flow volume (MEFV) curves, and determinations of closing volumes. The study population consisted of a total of 263 cases comprizing 124 cases of minimal, 78 moderatly and 61 far advanced pulmonary tuberculosis. On the whole, the mean of various volume and flow parameters derived from FEV and MEFV curves showed progressive decrease as the extent of the disease advanced. In comparisons of the mean of volume-adjusted flow parmeters between patients groups, all were significantly decreased in far advanced cases than that in the remainder, whereas significant differences between minimal and moderately advanced cases were limited to $\mathrm{FEV}_{1}, \dot{\mathrm{V}} \max 50$ and $\dot{\mathrm{V}} \max 25$. The mean of the closing volume $(\mathrm{CV})$ and its ratio to $\mathrm{VC}(\mathrm{CV} / \mathrm{VC})$ tended in far advanced cases was significantly larger than that in the remainder, with no significant difference between minimal and moderately advanced cases. This suggests that far advanced pulmonary tuberculosis is associated with a marked obstruction in large and small airways as well as restrictive ventilatory impairment. It also suggests that a major difference between minimal and moderately advanced cases is that the latter has a significant small airway obstruction compared with the former.
\end{abstract}

\section{緒 論}

근년에 와서 우수한 抗結核劑가 개발됨에 따라 結核의 치료가 용이하게 되었다. 그러나 우리나라에서는 아직도 肺結核환자가 적지 않으며 그로 인한 심한 換氣障碍나 肺 性心을 일으킨 예를 흔히 볼 수 있다. 肺結核에서 병변 이 진행 됨에 따라 肺氣腫 내지 閉塞性肺疾患이 병발할 수 있다는 것을 많은 학자들에 의해서 보고된 바가 있으 며 1 15) 본 교실에서도 肺結核의 肺機能에 관하여 여러차 례에 걸쳐 보고한 바가 있다 ${ }^{16 ~ 20)}$. 그러나 이 방면에 관 한 종전의 업적은 그 대다수가 呼吸計를 이용한 연구이 고, 근년에 많이 사용되고 있는 最大呼氣流量( $\mathrm{MEFV}$ ) 曲線의 분석이나 closing volume $(\mathrm{CV})$ 을 계측하여 大氣 道와 細小氣道의 기능을 분리해서 관찰한 것은 매우 드
물다. 그러나 $\mathrm{MEFV}$ 曲線에서 구하는 여러 지표들은 氣 道閉塞의 유무뿐 아니라 肺容量의 크고 적음에 따른 영 향을 받는다는 문제점이 있다. 그러므로 肺結核에서처럼 破壞性病變으로 인 한 制限性換氣障碍가 閉塞性換氣障碍 에 선행되는 질환에서는 $\mathrm{MEFV}$ 曲線에서 구한 呼氣流 速度를 반영하는 여러 지표들의 평가에 있어서 그것을 肺 容量에 대하여 교정해서 평가하는 것이 바람직하다 하겠 다 ${ }^{21)}$. 그러나 肺結核환자에서 구한 呼氣流速度를 肺容量 으로 교정해서 검토한 보고나 $\mathrm{CV}$ 를 관찰한 연구는 매우 드물며 더우기 우리나라에서는 아직 이 방면에 관한 업 적이 보고된 바가 없다.

저자들은 肺結核환자에서 肺機能을 평가하기 위한 연 구의 일환으로 이들 환자에서 기록한 努力性呼氣量 (FEV) 曲線 및 $\mathrm{MEFV}$ 曲線으로부터 계측한 呼氣流速 度를 반영하는 여러 지표들과 이들의 努力性肺活量 
$(\mathrm{FVC})$ 에 대한 교정치를 구하고 아울러 $\mathrm{CV}$ 를 측정하여 이들 성적을 肺結核의 重症度에 따라 서로 비교 검토하 고자 한다.

\section{對象 吕 方法}

肺結核환자 263 예 를 대상으로 하였으며 이들의 대부분 은 과거에 抗結核劑의 치료를 받은 적이 있거나 현재 치 료중인 환자들이었다. 肺結核의 重症度는 美國結核協會 의 분류 ${ }^{22}$ 에 따라 輕症, 中等症 및 重症으로 나누었다. 이들의 性別과 병변의 重症度는 Table 1 과 같이 輕症이 124예, 中等症 78예 重症이 61예였다. 그리고 여자는 전 예가 非吸煙者였으나 남자는 輕症, 中等症 및 重症에서 각각 65 예, 51 예 및 36 예가 吸煙者였다. 한편 이들의 年

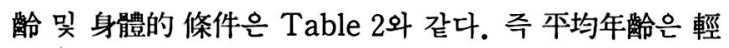
症에서 30.5세, 中等症에서 33.0세, 重症이 42.0세로서 重症度가 심해질수록 年鹷이 증가하는 경향이 있었고 平 均體重은 輕症에서 $56.3 \mathrm{~kg}$, 中等症에서 $54.0 \mathrm{~kg}$, 重症 에서 $49.3 \mathrm{~kg}$ 로서 重症度가 심할 수록 감소하였다.

그리고 $\mathrm{FEV}$ 曲線 및 $\mathrm{MEFV}$ 曲線은 Chest회사제 氣 流量曲線記錄計 OST-70으로 기록하였으며 그 구체적인 방법에 대해서는 앞서 본 교실의 趙등 ${ }^{23)}$ 이 상세히 기술 한 바가 있으나 그것을 요약하면 다음과 같다. 즉 $\mathrm{MEFV}$

Table 1. Sex and Extent of Disease in Patients with Pulmonary Tuberculosis

\begin{tabular}{lccc}
\hline \hline & Minimal & Moderate & Far \\
\hline Male & 107 & 66 & 49 \\
Female & 17 & 12 & 12 \\
\hline Total & 124 & 78 & 61 \\
\hline
\end{tabular}

Values are number of cases

In this and subsequent tables and figures minimal, moderate and far denote minimal, moderately and far advanced pulmonary tuberculosis, respectively
曲線의 기록은 피검자로 하여금 식후 2 시간 이상이 지난

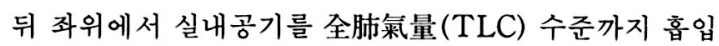
시킨 다음 되도록 빨리, 그리고 최대한으로 殘氣量 수준 까지 완전히 호출시키는 동안에 呼氣流速度는 $\mathrm{Y}$ 축에, 呼 氣量은 $\mathrm{X}$ 축에 동시에 기록하였다. 그리하여 이 곡선으 로부터 最高呼氣流速度 (peak expiratory flow rate, $\mathrm{PEFR}$ )와 $\mathrm{FVC}$ 의 $75 \%, 50 \%$ 및 $25 \%$ 에서의 最大呼氣 流速度, 즉 $\dot{\mathrm{V}} \max 75, \dot{\mathrm{V}} \max 50$ 및 $\dot{\mathrm{V}} \max 25$ 를 구하였 다. 또한 $\mathrm{MEFV}$ 曲線의 X軸의 아래에 동시에 그려지 는 $\mathrm{FEV}$ 曲線으로부터는 $\mathrm{FVC}$ 의 1 秒值 $\left(\mathrm{FEV}_{1}\right)$, 努力性 中間呼氣流速度(FEF 25-75\%), 그리고 $\mathrm{FVC}$ 의 1, 2 및 3 秒率 $\left(\mathrm{FEV}_{1} / \mathrm{FVC} \%, \mathrm{FEV}_{2} / \mathrm{FVC} \%\right.$ 및 $\mathrm{FEV}_{3} / \mathrm{FVC}$ $\%)$ 을 계측하였다.

한편 $\mathrm{CV}$ 는 氣流量曲線記錄計에 같은 Chest회사 제품 인 窒素分析計 STM-80N을 연결하여 Anthonisen등-4) 의 單回呼吸窒素法(single breath nitrogen mothod)에 준해서 측정하였으며 그 구체적인 방법은 앞서 본 교실 의 주등 ${ }^{25)}$ 이 기술한 바가 있으나 그것을 요약하면 다음 과 같다. 즉 피검자로 하여금 殘氣量 수준에서 $100 \% \mathrm{O}_{2}$ 를 송肺氣量 수준까지 홉입시킨 다음 피검자가 計器를 보 면서 呼氣를 초당 약 $0.5 \ell$ 의 속도로 殘氣量 수준까지 호 출해 내는 동안에 $\mathrm{N}_{2}$ 농도는 $\mathrm{Y}$ 軸에, 肺容量은 $\mathrm{X}$ 軸에 기 록하여 얻어지는 곡선에서 $\mathrm{N}_{2}$ 농도가 급격하게 상승하는 점에서 殘氣量 수준까지의 容量, 즉 $\mathrm{CV}$ 를 계측하였고 아 울러 $\mathrm{CV}$ 의 肺活量 $(\mathrm{CV})$ 에 대한 비, 즉 $\mathrm{CV} / \mathrm{VC}$ 를 산출 하였다.

그리고 모든 계측치는 推定正常值에 대한 백분율 $(\%)$ 로 표시하였는데 推定正常値를 산출하는 回歸方程式은 저자들의 교실에서 유도한 吸煙者 및 非吸煙者의 그것을 사용하였다 ${ }^{23,25 ~ 28)}$. 한편 $\mathrm{FEF} 25-75 \%, \mathrm{PEFR}, \dot{\mathrm{V}} \max$ $75, \dot{\mathrm{V}} \max 50$ 및 $\dot{\mathrm{V}} \max 25$ 의 실측치의 肺容量에 대한 교정치(volume-adjusted value)는 각 실측치를 $\mathrm{FVC}$ 로 나누어서 구하였다. 모든 통계검정은 Student $\mathrm{t}$ 검정과 $\mathrm{x}^{2}$ 검사를 사용하였으며 통계학적 유의성은 $\mathrm{p}<0.05$ (유

Table 2. Anthropometric Data of Patients with Pulmonary Tuberculosis

\begin{tabular}{llccc}
\hline \hline & Age (yrs.) & Ht (cm) & Wt (kg) & BSA (M $\left.{ }^{2}\right)$ \\
\hline Minimal & $30.5 \pm 12.88$ & $167.4 \pm 6.22$ & $56.3 \pm 7.24$ & $1.63 \pm 1.217$ \\
Moderate & $33.0 \pm 12.90$ & $167.0 \pm 6.98$ & $54.0 \pm 7.35$ & $1.59 \pm 0.126$ \\
Far & $42.0 \pm 14.07$ & $163.9 \pm 7.74$ & $49.3 \pm 7.80$ & $1.51 \pm 0.136$ \\
\hline
\end{tabular}

$\mathrm{Ht}=$ Height

$\mathrm{Wt}=$ Weight

BSA = Body surface area 
의수준)를 기준으로 하였다.

\section{成 績}

肺結核의 重症度에 따른 VC와 $\mathrm{FEV}$ 曲線에서 구한 여 러 지표의 계측치의 推定正常値에 대한 백분율의 성적은 Table 3 및 Fig. 1과 같다. 즉 거의 모든 지표들의 계측 치가 다 같이 비교적 심한 개인차가 있었다. 그러나 이 들의 평균을 보면 輕症에서는 $\mathrm{VC}$ 및 $\mathrm{FVC}$ 를 비롯하여 소위 時間肺活量(timed vital capacity, TVC) 즉 $\mathrm{FEV}_{1} / \mathrm{FVC} \%, \mathrm{FEV}_{2} / \mathrm{FVC} \%$, 및 $\mathrm{FEV}_{3} / \mathrm{FVC} \%$, 그리 고 $\mathrm{FEF} 25-75 \%$ 가 모두 推定正常值의 $90 \%$ 이상이었
다. 中等症에서는 $\mathrm{FEF} 25-75 \%$ 의 평균이 推定正常値 의 $74.9 \%$ 였던 것을 제외하면 나머지 지표들의 평균은 모 두 $80 \%$ 이상이었고 그 가운데서도 $\mathrm{FEV}_{1} / \mathrm{FVC} \%$, $\mathrm{FEV}_{2} / \mathrm{FVC} \%$, 및 $\mathrm{FEV}_{3} / \mathrm{FVC} \%$ 의 평균은 다 같이 90 $\%$ 이상이었다. 重症에서는 $\mathrm{VC}$ 가 $62.1 \%, \mathrm{FVC}$ 가 63.0 $\%, \mathrm{FEV}_{1}$ 이 $51.9 \%$ 로 비교적 심한 감소를 보였고 $\mathrm{FEV}_{1} / \mathrm{FVC} \%, \mathrm{FEV}_{2} / \mathrm{FVC} \%$, 및 $\mathrm{FEV}_{3} / \mathrm{FVC}^{2}$ 는 각 각 $78.0 \%, 84.2 \%, 89.0 \%$ 로서 약간의 감소, 그리고 $\mathrm{FEF} 25-75 \%$ 는 $37.8 \%$ 로서 매우 심한 감소를 보였다. 따라서 重症에서는 $\mathrm{FEV}_{2} / \mathrm{FVC} \%$ 및 $\mathrm{FEV}_{3} / \mathrm{FVC} \%$ 를 제외한 모든 계측치가 다 같이 $80 \%$ 이하로 감소되어 있 었다. 그리하여 $\mathrm{VC}, \mathrm{FVC}, \mathrm{FEV}_{1}, \mathrm{FEV}_{1} / \mathrm{FVC} \%$ 및

Table 3. Parameters Derived from Forced Expiratory Flow Volume Curves in Patients with Pulmonary Tuberculosis

\begin{tabular}{lccc}
\hline & Minimal $(\mathrm{n}=124)$ & Moderate $(\mathrm{n}=78)$ & Far $(\mathrm{n}=61)$ \\
\hline VC & $91.3 \pm 15.06$ & $82.9 \pm 15.50$ & $62.1 \pm 18.00$ \\
$\mathrm{FVC}$ & $92.6 \pm 14.07$ & $86.7 \pm 15.95$ & $63.0 \pm 15.99$ \\
$\mathrm{FEV}_{1}$ & $91.7 \pm 19.72$ & $80.8 \pm 20.49$ & $51.9 \pm 22.03$ \\
$\mathrm{FEV}_{1} / \mathrm{FVC} \%$ & $96.5 \pm 13.20$ & $91.5 \pm 15.48$ & $78.0 \pm 19.00$ \\
$\mathrm{FEV}_{2} / \mathrm{FVC} \%$ & $97.7 \pm 8.41$ & $96.2 \pm 8.74$ & $84.2 \pm 14.82$ \\
$\mathrm{FEV}_{3} / \mathrm{FVC} \%$ & $98.8 \pm 6.46$ & $97.4 \pm 6.21$ & $89.0 \pm 12.41$ \\
$\mathrm{FEF}_{2}$ - 75\% & $91.2 \pm 37.86$ & $74.9 \pm 33.20$ & $37.8 \pm 30.71$ \\
\hline
\end{tabular}

In this and subsequent tables, values are mean \pm S.D. expressed as a percentage of the predicted.

$\mathrm{VC}=$ Vital capacity

FVC $=$ Forced vital capacity

$\mathrm{FEV}_{1}=$ Forced expiratory volume in one second

$\mathrm{FEV}_{1},{ }_{2} \&{ }_{3} / \mathrm{FVC} \%=$ Percentage forced expiratory volume in one, two and three seconds

FEF $25-75 \%=$ Maximal mid-expiratory flow

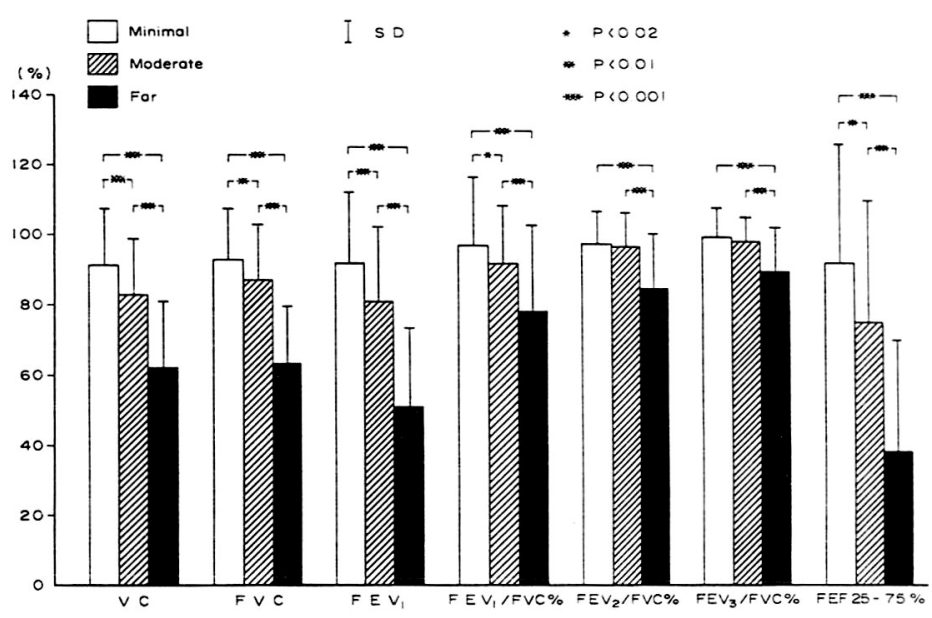

Fig. 1. Comparisons of vital capacity and parameters derived from forced expiratory volume curve by the extent of disease in pulmonary tuberculosis. 
$\mathrm{FEF} 25-75 \%$ 의 평균은 병변의 重症度에 따른 유의한 감소, 즉 中等症은 輕症에 비하여, 그리고 重症은 中等 症에 비하여 유의한 감소를 보였다. 그러나 $\mathrm{FEV}_{2} / \mathrm{FVC}$ $\%$ 및 $\mathrm{FEV}_{3} / \mathrm{FVC} \%$ 는 輕症과 中等症 사이에는 유의한 차이가 없고 重症과 輕症 및 中等症사이에만 유의한 감 소가 있었다.

그리고 $\mathrm{MEFV}$ 曲線에서 구한 여러 지표의 推定正常 值에 대한 백분율의 성적은 Table 4 및 Fig. 2 와 같이 이 들 지표의 계측치 역시 모두가 상당한 개인차를 보였다. 이들의 평균치를 보면 輕症에서는 PEFR, $\dot{\mathrm{V}} \max 75$, $\dot{\mathrm{V}} \max 50$ 그리고 $\dot{\mathrm{V}} \max 25$ 가 모두 推定正常值의 $80 \%$ 이상이었고 $\dot{V} \max 75$ 는 $90 \%$ 이상이었다. 中等症에서 는 모두 推定正常値의 $80 \%$ 이하였고 $\dot{\mathrm{V}} \max 50$ 은 67.3 $\%$ 로 $70 \%$ 미만이었다. 重症에서는 PEFR의 평균이 약 $50 \%$ 였고 $\dot{\mathrm{V}} \max 75, \dot{\mathrm{V}} \max 50$ 및 $\dot{\mathrm{V}} \max 25$ 가 모두 40 $\%$ 미만이었다. 병변의 범위에 따른 이들 지표의 증감의

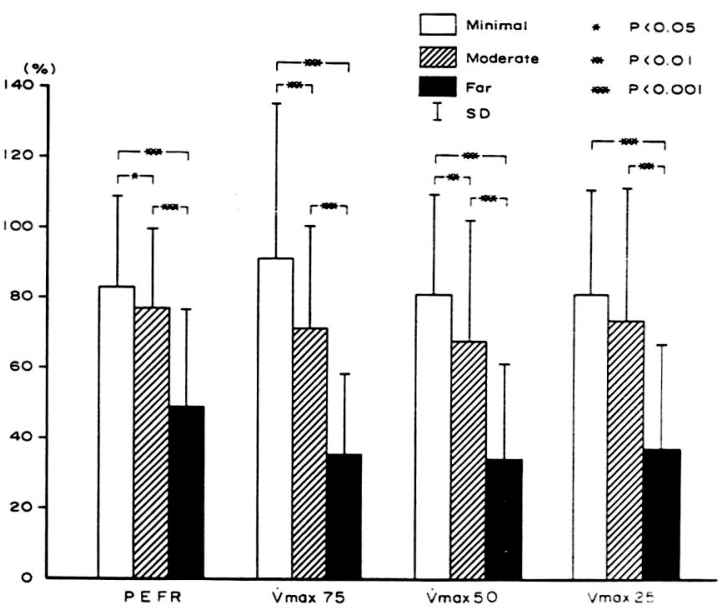

Fig. 2. Comparisons of parameters derived from maximal expiratory flow volume curve by the extent of disease in pulmonary tuberculosis.
정도는 PEFR, $\dot{\mathrm{V}} \max 75, \dot{\mathrm{V}} \max 50$ 및 $\dot{\mathrm{V}} \max 25$ 가 모 두 병변의 범위가 심할 수록 그 감소의 정도도 심하였으 며 그 가운데서도 PEFR, $\dot{\mathrm{V}} \max 75, \dot{\mathrm{V}} \max 50$ 및 $\dot{\mathrm{V}} \max 25$ 은 병변의 범위에 따른 차이가 모두 유의하였 으며 $\operatorname{Vmax} 25$ 는 中等症과 重症, 輕症과 重症사이에 유 의하였다.

한편 $\mathrm{CV}$ 및 $\mathrm{CV} / \mathrm{VC}$ 는 Table 5 및 Fig. 3 과 같이 개 개의 계측치는 역시 개인차가 심하였다. 그리고 呼氣의 肺內分布障碍가 심해서 $\mathrm{CV}$ 의 계측이 불가능했던 예가 中 等症에서 7예, 重症에서 1예 있었는데, 이들을 합해서 $\mathrm{CV}$ 를 계측하지 않았던 예를 제외한 나머지 218 예에서의 $\mathrm{CV}$ 의 평균을 보면 병변의 정도가 심해짐에 따라 증가하 는 경향을 보였으나 이들 차이는 輕症과 重症사이의 차 이만이 유의하였다. 그리고 $\mathrm{CV} / \mathrm{VC}$ 도 $\mathrm{CV}$ 와 마찬가지 로 병변의 정도가 심해짐에 따라 증가하였으며 輕症과 重 症, 그리고 中等症과 重症사이의 차이는 유의하였다.

한편 呼氣流速度를 나타내는 여러 계측치가운데 FVC 의 영향을 받는 지표들의 容量矯正值의 성적을 보면

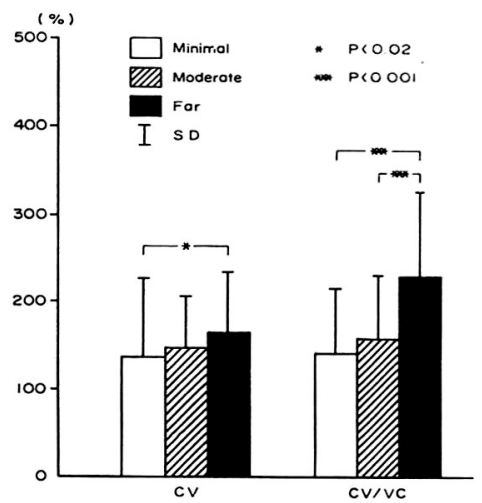

Fig. 3. Coinparisons of closing volumes by the extent of disease in pulmonary tuberculosis.

Table 4. Parameters Derived form Maximal Expiratory Flow Volume Curves in Patients with Pulmonary Tuberculosis

\begin{tabular}{lccr}
\hline \hline & Minimal $(\mathrm{n}=124)$ & Moderate $(\mathrm{n}=78)$ & Far $(\mathrm{n}=61)$ \\
\hline PEFR & $83.1 \pm 20.72$ & $76.7 \pm 22.60$ & $49.1 \pm 23.50$ \\
$\dot{V} \max 75$ & $90.9 \pm 44.06$ & $71.2 \pm 28.49$ & $35.8 \pm 26.09$ \\
$\dot{\mathrm{V} m a x} 50$ & $80.6 \pm 29.34$ & $67.3 \pm 33.20$ & $34.4 \pm 25.68$ \\
$\dot{V} \max 25$ & $80.4 \pm 26.93$ & $73.3 \pm 37.36$ & $36.9 \pm 31.66$ \\
$\dot{\mathrm{V}} \max 50 / 25$ & $92.1 \pm 23.78$ & $94.1 \pm 22.96$ & $105.8 \pm 40.37$ \\
\hline
\end{tabular}

PEFR $=$ Peak expiratory flow rate

$\dot{V} \max 75,50 \& 25=$ Maximal expiratory flow rate at $75,50 \& 25 \%$ of forced vital capacity 
Table 5. Closing Volume (CV) and its Ratio to Vital Capacity (CV/VC)

\begin{tabular}{lccc}
\hline \hline & Minimal (n=103) & Moderate $(\mathrm{n}=64)$ & Far $(\mathrm{n}=51)$ \\
\hline $\mathrm{CV}$ & $135.8 \pm 67.70$ & $147.7 \pm 54.10$ & $163.1 \pm 63.30$ \\
$\mathrm{CV} / \mathrm{VC}$ & $141.2 \pm 73.03$ & $158.5 \pm 63.88$ & $228.9 \pm 98.80$ \\
\hline $\mathrm{CV}=$ Closing volume & $\mathrm{VC}=$ Vital capacity & $\mathrm{CV} / \mathrm{VC}=$ Ratio of CV to VC
\end{tabular}

Table 6. Forced Mid-expiratory Flow and Parameters Derived from Maximal Expiratory Flow Volume Curves Adjusted for Volume in Patients with Pulmonary Tuberculosis

\begin{tabular}{lccc}
\hline \hline & Minimal $(\mathrm{n}=124)$ & Moderate $(\mathrm{n}=78)$ & Far $(\mathrm{n}=61)$ \\
\hline FEF 25 - 75 \%/FVC & $0.98 \pm 0.395$ & $0.87 \pm 0.422$ & $0.48 \pm 0.416$ \\
PEFR/FVC & $2.04 \pm 0.469$ & $2.01 \pm 0.535$ & $1.72 \pm 0.790$ \\
$\dot{V} \max 75 /$ FVC & $1.70 \pm 0.528$ & $1.57 \pm 0.631$ & $1.06 \pm 0.768$ \\
$\dot{\operatorname{Vmax}}$ 50/FVC & $1.06 \pm 0.403$ & $0.91 \pm 0.449$ & $0.53 \pm 0.458$ \\
$\dot{V} \max$ 25/FVC & $0.54 \pm 0.345$ & $0.41 \pm 0.246$ & $0.20 \pm 0.228$ \\
\hline
\end{tabular}

Values are expressed in liter/sec/liter

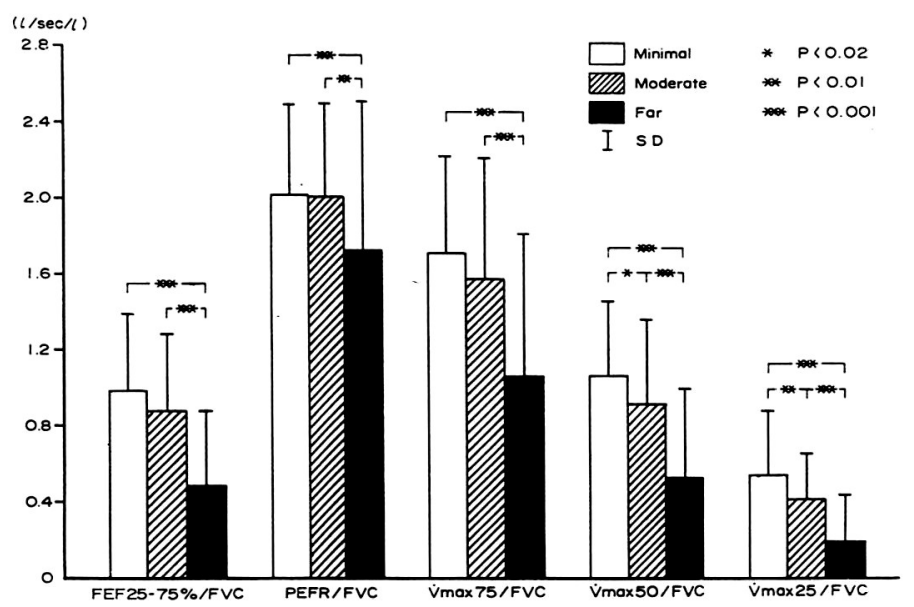

Fig. 4. Comparisons of forced mid-expiratory flow and parameters derived from maximal expiratory flow volume curves, adjusted for volume, by the extent of disease in pulmonary tuberculosis.

Table 6 및 Fig. 4와 같이 非矯正值, 즉 실측치와 마찬 가지로 병변의 정도가 심할 수록 감소하는 경향이 있었 다. 그러나 FEF 25-75\%/FVC, PEFR/FVC, $\dot{\mathrm{V}} \max$ $75 / \mathrm{FVC}$ 는 輕症과 中等症사이의 차이가 유의하지 않았 으며 $\dot{\mathrm{V}} \max 50 / \mathrm{FVC}$ 및 $\dot{\mathrm{V}} \max 25 / \mathrm{FVC}$ 는 양군사이의 차이가 유의하였다. 그리고 重症에서는 容量矯正值가 모 두 輕症 및 中等症에 비해서 유의한 감소를 보였다.

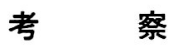

肺結核환자의 換氣機能은 병변의 범위와 성상, 부위, 이환기간등을 비롯하여 병변에 속발되는 이차적 변화에 따라 차이가 있을 것으로 생각되나 結核과 같은 다양한 경과를 취하는 만성병에서는 이차적으로 발생하는 변화 도 다양하기 때문에, 그 결과로서 초래되는 換氣障碍의 양상에도 상당한 차이가 있을 것으로 생각된다. 일반적 으로 肺組織데 結核性病變이 발생하면 일차적으로는 制 
限性換氣障碍가 나타날 것이나 結核性病變에 속발하는 肺纖維化, 氣道의 병변이나 㷋窄 또는 擴張, 肋膜癒着, 病變部의 肺容量의 감소와 그것에 따른 건강한 肺組織의 過膨張 내지 肺氣腫, 縱隔洞의 이동등은 氣道閉塞을 일 으켜서 閉塞性換氣障碍도 초래될 수 있을 것으로 예상된 다. 즉 Bell ${ }^{29}$ 은 肺에 結核感梁을 일으킨 토끼에서 肺氣 腫이 발생함을 관찰하였고 그 밖에 여러 학자들도 $8,11,30$ 32) 肺結核환자에서 病變이 진행 됨에 따라 肺氣腫 또는 閉塞 性肺疾患의 발생 빈도가 높아짐을 病理學的으로 또는 肺 機能검사에 의해서 입증하였다. 한편 Snider등5)은 肺結 核환자에서 慢性氣管枝炎, 肺氣腫 또는 氣管枝喘息은 肺 結核과 관계없이 우연히 동반되어서 나타나는 경우도 있 으나 氣管枝擴張은 병변의 정도가 심하거나 유병기간이 길 수록 그 빈도가 높다고 하였다. 그리고 일찌기 Hutchinson ${ }^{33)}$ 이 肺結核에서는 VC가 감소함을 보고한 이래 肺結核환자의 換氣機能에 대해서는 많은 연구가 있 다. 즉 Simpson등 4)은 肺結核의 호전에 따라 $\mathrm{VC}$ 가 꾸 준히 증가하며 그 증가는 특히 초기에 현저하다고 하였 고 Malik 및 $\operatorname{Martin}^{13)}$ 은 병변의 호전에 따는 $\mathrm{VC}$, 最 大換氣量, puffmeter ${ }^{34)}$ 로 측정한 最大呼氣流速度 (maximal expiratory flow rate, MEFR), 그리고 擴 散能의 변동을 관찰하였던바 $\mathrm{VC}$ 를 제외한 지표들은 병 변이 호전되어도 영향을 받지 않았다고 하였다. 그러나 Willians등 ${ }^{35)}$ 은 肺의 擴散能과 병변의 범위와는 관계가 있으나 VC와 병변의 범위와는 관계가 없다고 하였다. 또 한 Martin 및 $\mathrm{Hallet}^{9)}$ 은 710 명의 肺綰核환자의 $34 \%$ 에 서 閉塞性肺疾患이 있었고 VC는 MEFR과 상당한 상관 관계가 있으며 $\mathrm{VC}$ 가 $2 \ell$ 이상인 경우에는 $\mathrm{MEFR}$ 이 정 상이며 $\mathrm{VC}$ 가 $2 \ell$ 이하일 때는 氣道閉塞이 있다고 하였 다. 한편 Anno 및 Tomashefski ${ }^{11)}$ 는 肺結核에서는 이 차적으로 肺氣腫이 발생할 수 있으며 肺氮腫의 유무는 $\mathrm{VC}$ 보다는 最大換氣量의 감소에 더 잘 반영된다고 하였 다. 또한 朴 $^{16)}$ 은 $\mathrm{VC}$ 는 대체로 병변의 범위에 일치해서 감소하나 輕症과 中等症사이의 차이는 경미하고 中等症 과 重症사이에는 현저한 차이가 있는데 重症 혹은 中等 症 가운데도 $\mathrm{VC}$ 가 정상인 예가 다수 있어서 $\mathrm{VC}$ 만으로 는 병변의 경중을 판단할 수는 없다고 하였다. 그리고 朴 ${ }^{16)}$ 은 輕症과 中等症사이에 $\mathrm{VC}$ 에 큰 차이가 없는 것은 병변의 범위보다는 $\mathrm{VC}$ 에 크게 영향을 미치지 않는 작은 空洞의 유무가 양자간의 주되는 차이인 경우도 비교적 많 았기 때문이라 하였다. 저자들의 성적에서는 輕症에서는
$\mathrm{VC}$ 의 평균이 推定正常值의 $91.3 \%$ 로서 정상보다 약간 감소되어 있음에 불과하였으나 병변의 정도가 심해질수 록 뚜렷하게 감소하였으며 이러한 성적은 Williams등 ${ }^{35)}$ 의 성적과는 상반되나 Hutchinson ${ }^{33)}$ 및 朴 $^{16)}$ 의 성적과 는 일치한다.

肺結核환자의 $\mathrm{FVC}, \mathrm{FEV}_{1}$ 및 $\mathrm{TVC}$ 에 대해서는 여러 보고가 있으나 그 성적에는 약간의 차이가 있다. 즉 肺 結核에서 $\mathrm{FVC}, \mathrm{FEV}_{1}$ 또는 $\mathrm{FEV}_{1} / \mathrm{FVC} \%$ 등의 계측치 가 감소한다는 사실에 대해서는 이론이 없으나 그 감소 의 정도나 병변의 범위와 감소의 정도와의 관계에 대해 서는 보고자에 따라서 다소의 차이가 있다 ${ }^{5 \sim 7,16,32)}$. 그러 나 일반적으로 합병증이 없는 輕症에서는 呼氣流의 閉塞 이 없으며 오래된 活動性肺結核에서는 氣道閉塞이 심해 지는 것으로 ${ }^{2)}$ 알려져 있다. 또한 $\mathrm{Ahn}$ 등ㄱ)은 감염된 抗 酸菌의 종류에 따라 換氣機能에도 차이가 있다고 하였는 데 人型菌에 의한 輕症에서는 $\mathrm{FVC}$ 의 평균이 推定正常 值의 $93.2 \%$ 로서 정상범위안에 있고 병변의 범위가 증가 함에 따라 감소한다고 하였다. 그리고 $\mathrm{Ahn}$ 등 7$)$ 은 輕症 에서는 $\mathrm{FEV}_{1} / \mathrm{FVC} \%$ 의 推定正常値의 평균이 $76.8 \%$ 로 서 정상범위에 가까왔으며 병변의 정도가 심해짐에 따라 감소한다 하였다.

그러나 Mycobaicterium intracellulare에 의한 輕症 肺結核에서는 $\mathrm{FVC}$ 의 평균이 推定正常値의 $73.1 \%$ 밖에 되지 않았는데 그 이유는 M. intracellulare는 肋膜癒着, 氣管支擴張, 肺纎維化등을 흔히 동반하기 때문이라 하였 다. 또한 Snider등 ${ }^{5}$ 은 1403 명의 肺結核환자에서 $\mathrm{FEV}_{1}$ 이 推定正常値의 $80 \%$ 이하인 예가 $60 \%$ 였고 $\mathrm{FEV}_{1} / \mathrm{VC}$ $\%$ 가 $70 \%$ 이하인 예가 $42 \%$ 였다고 하였다. 그리고 훙부 $\mathrm{X}$ 선상의 병변의 정도와 $\mathrm{VC}, \mathrm{FEV}_{1}$, 및 $\mathrm{FEV}_{1} / \mathrm{VC} \%$ 와 의 관계를 보았던 바 이들 사이에 약간의 역상관관계가 있었으며 그 가운데서도 $\mathrm{VC}$ 와 더욱 그러하였다고 하였 다. 또한 Krishna등히은 276 명의 환자를 추적검사하였던 바 $\mathrm{FEV}_{1}$ 과 $\mathrm{FVC}$ 가 원래의 병변의 범위, 그리고 肺䌭維 化, 肺氣腫, 肋膜肥厚의 유무와 상당한 역상관관계가 있 었는데, 특히 $\mathrm{FEV}_{1}$ 이 그러하였으며 $\mathrm{FEV}_{1}$ 에 많은 영향 을 미치는 인자는 肺纖維化라 하였다. 한편 Curtis등 ${ }^{32}$ 은 肺結核에서 肺氣腫의 유무의 기준으로 $\mathrm{FEV}_{1} / \mathrm{FVC} \%$ 는 $73 \%, \mathrm{FEV}_{2} / \mathrm{FVC} \%$ 는 $89 \%, \mathrm{FEV}_{3} / \mathrm{FVC} \%$ 는 93.1 $\%$ 로 정했으며 이 가운데서도 $\mathrm{FEV}_{2} / \mathrm{FVC} \%$ 가 가장 좋 은 기준이 된다고 하였다. 그리고 朴 $^{16)}$ 은 肺結核에서 $\mathrm{FEV}_{1} / \mathrm{FVC} \%$ 가 重症에서는 약간 감소되었으나 輕症과 
中等症의 성적은 대체로 건강인과 비슷하였고 $\mathrm{FEV}_{2}$ / $\mathrm{FVC} \%$ 및 $\mathrm{FEV}_{3} / \mathrm{FVC}^{2}$ 는 건강인에 비해서 감소하는 경향이 있었으나 그 정도는 重症에서 더욱 심하다고 하 였으며 重症에서 TVC의 저하가 있는 것은 Anno 및 Tomaschefski $\mathbf{i}^{11}$ 가 지적한 바와 같이 병변의 진행에 따 라 肺氣腫의 발생빈도가 높기 때문일 것이라고 하였다. 저자들의 성적에서 輕症에서는 $\mathrm{FVC}$ 의 평균이 推定正常 值의 $92.6 \%$ 로서 $\mathrm{Ahn}$ 등 7$)$ 의 성적과 비슷하였고 또한 병 변의 진행에 따라 유의하게 감소하였다. 그러나 $\mathrm{FEV}_{1} /$ $\mathrm{FVC} \%$ 의 평균은 $96.5 \%$ 로서 $\mathrm{Ahn}$ 등 7 의 성적에 비해서 다소 높았으나 다른 보고들5 7,16)에서와 마찬가지로 병변 이 진행함에 따라 유의하게 감소하였다.

한편 肺結核환자의 FVC 曲線에서 구한 FEF 25-75 $\%$ 에 대하여 Snider등 ${ }^{1}$ 은 1403 명의 환자가운데 $43 \%$ 에 서 $100 \mathrm{\ell} / \mathrm{min}$ 이하였다고 하였으며 Marcus등 ${ }^{36)}$ 은 100 명의 환자를 6 개월간 추적검사 하였던 바 치료전에는 평 균 $1.8 \mathrm{\ell} / \mathrm{sec}$ 였고 치료후에는 $2.3 \mathrm{l} / \mathrm{sec}$ 로서 약간의 증 가가 있었으나 유의한 차이는 없었다고 하였다. 또한 $\mathrm{Ahn}$ 등 ${ }^{7)}$ 은 232 명의 환자에서 $\mathrm{FEF} 25-75 \%$ 의 평균치는 $123 \ell / \mathrm{min}$ 로서 推定正常値의 $60.3 \%$ 였으나 이 가운데 36 명의 輕症에서는 $\mathrm{FEF} 25-75 \%$ 가 $78.4 \%$ 로서 정상범위 내에 있었으며 $\mathrm{FEF} 25-75 \%$ 는 $\mathrm{FEV}_{1} / \mathrm{FVC} \%$ 보다 병 변의 범위에 따른 감소가 뚜렷하다고 하였다. 저자들의 성적에서는 輕症에서 $\mathrm{FEF} 25-75 \%$ 의 평균이 推定正常 值의 $91.2 \%$ 로서 $\mathrm{Ahn}$ 등ㄱ)의 성적보다는 높았으나 병변 이 진행함에 따라 현저한 감소를 보인것은 서로 일치하 는 소견이라 하겠다.

한편 閉塞性肺疾患의 진단에 종전에는 소위 TVC, 氣 速係數 (air velocity index) 및 FEF 25-75\%등의 지표 가 주로 사용되었으나 근년에는 $\mathrm{MEFV}$ 曲線의 분석이 더욱 예민한 진단방법으로 알려져 있다. 즉 이 曲線에서 구하는 여러 지표들 가운데 PEFR 및 $\dot{\mathrm{V}} \max 75$ 는 $\mathrm{FEV}_{1}$ 처럼 주로 大氣道機能을 반영하고 $\dot{\mathrm{V}} \max 50$ 은 주로 細 小氣道機能을 반영하나 中氣道機能 내지 大氣道機能도 포함되며 $\dot{V} \max 25$ 는 細小氣道機能을 반영한다고 한 다 $\left.{ }^{37} 39\right)$. 그러나 이들 지표에 반영되는 大氣道 및 細小 氣道機能은 서로 상관이 있기 때문에 이들 양자를 엄격 하게 분리해서 측정하는 것은 어려운 것으로 알려져 있 다. 또한 呼氣流速度를 반영하는 지표들은 $\mathrm{VC}$ 의 감소에 의해서도 그 값이 감소한다 ${ }^{21,40)}$.

肺結核 환 자 의 maximum expiratory flow rate
(MEFR)에 관한 보고는 드물며 $\mathrm{MEFV}$ 曲線의 분석에 관한 업적은 거의 없는 듯 하다. Hallet 및 $\mathrm{Martin}^{8)}$ 은 肺結核환자에서 병변의 범위에 다른 MEFR의 변화를 관 찰하였던 바 輕症에서는 평균이 $295 \mathrm{l} / \mathrm{min}$ 로서 $22 \%$ 에 서 呼氣流速度의 감소가 있었고 中等症에서는 $270 \mathrm{l} / \mathrm{min}$ 로서 $57 \%$ 에서 呼氣流速度가 감소되어 있었으며 MEFR 과 병변의 범위와는 상당한 관계가 있었다고 하였다.

또한 Martin 및 Hallet ${ }^{9)}$ 는 710명의 肺結核가운데 15 $\%$ 에서는 MEFR이 $160 \ell / \mathrm{min}$ 이상이었으나 건강인 에서 보다는 낮으므로써 경한 氣道閉塞이 있음을 나타내었고 $11 \%$ 에서는 중등도의 氣道閉塞이, 그리고 $8 \%$ 에서는 $\mathrm{MEFR}$ 이 $110 \mathrm{\ell} / \mathrm{min}$ 이하로 심한 氣道閉塞이 있었다고 하였으며 MEFR은 最大換氣量과 상관관계가 좋다고 하 였다. 그리고 Martin 및 Hallet ${ }^{9)}$ 는 MEFR과 呼吸困難 과는 역 상관관계가 있고 홍부X-선 변화와도 중등도의 역 상관관계가 있다고 하였다. 또한 Martin 및 Hallet ${ }^{10)}$ 는 輕症과 中等症에서는 MEFR이 매년 $2.43 \ell$, 重症에서 는 매년 $1.3 \ell$ 감소하며 輕症과 中等症에서는 MEFR의 감소의 정도와 연령과의 사이에 관련이 있었으나 重症에 서는 관련이 없었다고 하였다. 그리고 Martin등1)은 肺 結核의 진행정도와 最大換氣量, MEFR과는 관계가 있 으며 輕한 氣道閉塞이 있는 환자군에서는 심한 氣道閉塞 이 있는 환자군에서 보다 重症肺結核이 횔씬 적었다고 하 였으며 MEFR이 $105 \ell / \mathrm{min}$ 이하, 最大換氣量이 推定正 常值의 $40 \%$ 미만인 심한 氣道閉塞이 있는 환자의 $50 \%$ 가 重症結核이었다고 하였다. 또한 Pardee등'2)은 活動性 肺結核의 지속기간이 길고 심할수록 氣道閉塞이 발생하 기 쉽다고 하였다. 저자들의 성적에서 PEFR은 병변이 심할수록 현저한 감소를 보였고 특히 重症에서 그러하였 으며 $\dot{\mathrm{V}} \max 75, \dot{\mathrm{V}} \max 50$ 및 $\dot{\mathrm{V}} \max 25$ 는 역시 비슷한 경향의 변화를 보였다. 이러한 소견은 병변이 진행할 수 록 肺實質의 파괴에 따른 이차적인 변화가 大氣道 및 細 小氣道의 閉塞을 더욱 심하게 일으키게되기 때문인 것으 로 생각된다.

細小氣道의 機能障碍의 조기진단에는 여러가지 방법이 이용되고 있으며 ${ }^{37,41 ~ 45)} \mathrm{CV}$ 의 계 측도 그 가운데의 하나 이다. $\mathrm{Macklem}^{46)}$ 에 의하면 $\mathrm{CV}$ 의 측정은 容壓率의 呼 吸數依存性 (frequency dependence of compliance) 만 큼 예민하게 細小氣道의 변화를 반영하고 또한 $\mathrm{FEF}$ 25-75\%보다 약 2배나 예민하다고 한다. 肺結核에서의 $\mathrm{CV}$ 에 관한 보고는 매 우 드물며 Curtis등 ${ }^{32)}$ 그리고 Curtis 
및 Rassmusen ${ }^{47}$ 은 肺結核에서 單回呼吸酸素法을 이용 하여 呼氣終末氣에 $\mathrm{N}_{2}$ 농도가 상승하는 것을 관찰함으로 서 換氣分布障碍에 대한 유용한 정보를 얻을 수 있다고 하였으며 肺氣腫이 합병된 경우에 $\mathrm{N}_{2}$ 농도의 이상상승은 肺氣腫이 합병되지 않고 空洞, 肺胞性肺囊腫, 氣管枝擴 張症, 肋膜肥厚 또는 橫隔膜의 운동장애등이 있어도 나 타나기 때문에 $\mathrm{N}_{2}$ 농도의 상승만으로 肺氣腫의 유무를 판 단하기 어려운 경우도 있다고 하였다. 저자들의 성적에 서는 $\mathrm{CV}$ 는 병변의 정도가 심할수록 증가하는 경향이 있 었고 輕症과 重症사이의 차이는 유의하였다. 한편 $\mathrm{CV} /$ $\mathrm{VC}$ 는 輕症과 中等症사이에는 유의한 차이가 없었으나 中 等症과 重症, 그리고 輕症과 重症사이에는 유의한 차이 가 있었으며 이것은 병변의 정도가 심할 수록 $\mathrm{CV}$ 는 증 가하는 경향이 있었고 $\mathrm{VC}$ 는 감소했기 때문인 것으로 생 각된다. 그리고 $\mathrm{CV}$ 및 $\mathrm{CV} / \mathrm{VC}$ 의 이러한 성적은 肺結 核에서는 細小氣道의 閉塞이 나타나며 특히 重症에서 그 러함을 시사한다.

한편 氣道病變이 매우 심한 예에서는 $\mathrm{CV}$ 의 측정이 불 가능한 경우도 있다. 즉 McCarthy등43)은 46명의 吸煙 者에서 argon을 이용하여 $\mathrm{CV}$ 의 계측을 시도하였는데 1 예에서는 單回呼氣曲線에서 argon농도가 계속적으로 심 히 상승하여 $\mathrm{CV}$ 를 계측할 수 없었다 하고 이것은 심한 換氣分布障碍 때문이라 하였다. 또한 helium을 이용한 Green등 ${ }^{48)}$ 은 낮은 肺容量位에서 單回呼氣曲線에 心博動 에 의한 심한 진동 (cardiac oscillation)이 나타나거나 혹 은 alveolar plateau가 평탄하지 않을때도 $\mathrm{CV}$ 를 계측할 수 없다고 하였다. 저자들의 관찰에서도 呼氣時의 換氣 分布障碍가 심해서 $\mathrm{CV}$ 의 측정이 불가능하였던 예는 中 等症 64예가운데 7예, 重症 51예가운데 1예가 있었다.

한편 MEFV 曲線에서 구한 呼氣流速度를 반영 하는 지 표들의 실측치는 肺容量의 변동에 따라 차이가 있을 수 있으므로 ${ }^{21,40,49,50)}$. 이러한 차이를 줄이기 위하여 이들 지 표의 실측치를 VC, FVC 또는 TLC로 교정해서 표시 하는 것이 바람직하다 하겠다. Zapletal등 ${ }^{49)}$ 은 6 18세 의 연령군을 대상으로 呼氣流速度를 반영하는 여러 지표 를 TLC로 교정 ( $\dot{\mathrm{V}} \max / \mathrm{TLC})$ 해 보았던 바, 身長의 크 고 적음에 따른 이들 지표의 차이를 감소시킬 수 있었다 고 하였다. 한편 Jayamanne등 21)은 彌滿性間質性肺疾患 에서 PEFR은 氣道抵抗이 정상인 경우에는 肺容量과 정 상관관계가 있고 또한 $\dot{\mathrm{V}} \max 50$ 및 $\dot{\mathrm{V}} \max 25$ 는 그 실 측치가 정상 혹은 그 이하인 경우에는 이들을 TLC로 교
정하면 그 값이 정상 혹은 그 이상이 됨을 관찰하였다. 그리하여 이들의 실측치는 容量依存性(volume dependent)이며 非矯正值의 감소는 氣道疾患을 의미하는 것이 아닐 수 있을 것이라고 하고 肺容量의 감소가 있는 경우 에는 呼氣流速度를 반영하는 지표의 평가에 있어서 이들 의 실측치를 肺容量에 대해서 교정해야만 할 것이라 하 였다. 또한 Clement 및 Woestijne ${ }^{40}$ 도 동일인에서 $\mathrm{VC}$ 의 차이가 呼氣流速度에 차이를 나타낼 수 있다고 하였 다. 저자들은 呼氣流速度를 반영하는 지표들의 실측치의 肺容量에 대한 교정을 $\mathrm{FVC}$ 로 하였는데 $\dot{\mathrm{V}} \max 50 / \mathrm{FVC}$, $\dot{\mathrm{V}} \max 25 / \mathrm{FVC}$ 및 $\mathrm{FEV}_{1} / \mathrm{FVC} \%$ 는 輕症과 中等症사이 에 유의한 감소가 있었는 반면 $\mathrm{FEF} 25-75 \% / \mathrm{FVC}$, $\mathrm{PEFR} / \mathrm{FVC}, \dot{\mathrm{V}} \max 75 / \mathrm{FVC}, \quad \mathrm{FEV}_{2} / \mathrm{FVC} \%$ 및 $\mathrm{FEV}_{3} / \mathrm{FVC} \%$ 의 輕症과 中等症사이의 차이는 유의하지 않았다. 그러나 重症에서는 이들 모든 지표가 다같이 中 等症에서 보다 유의하게 감소되어 있었다.

이러한 소견은 輕症과 中等症사이에는 병변이 진행 함 에 따라 주로 細小氣道의 閉塞이 나타나게되나 병변이 더 욱 진행함에 따라 細小氣道의 閉塞뿐만 아니라 大氣道의 閉塞도 동반됨을 나타내는 것이라 하겠다.

\section{結論}

肺結核환자에서 병변의 범위에 따른 換氣力學의 차이 를 알아보기 위하여 輕症 124예, 中等症 78예, 重症 61 예에서 $\mathrm{VC}$ 를 비롯하여 $\mathrm{FEV}$ 曲線 및 $\mathrm{MEFV}$ 曲線에서 구한 여러 지표의 실측치와 그들의 容量矯正値, 그리고 $\mathrm{CV}$ 및 $\mathrm{CV} / \mathrm{VC}$ 를 병변의 경중에 다라 서로 비교 검토 하여 다음과 같은 성적을 얻었다.

肺結核에서는 VC 및 FVC를 비롯하여 FVC 曲線 및 $\mathrm{MEFV}$ 曲線에서 구한 呼氣流速度를 반영하는 여러 지 표들 즉 $\mathrm{FEV}_{1}, \dot{\mathrm{V}} \max 75, \dot{\mathrm{V}} \max 50, \dot{\mathrm{V}} \max 25$ 의 여 러 실측치 및 그들의 $\mathrm{FVC}$ 에 대한 容量矯正値가 모두 병 변의 정도가 심할수록 감소하는 경향이 있었으며 重症에 서는 TVC를 포함한 이들 지표의 모든 容量矯正値가 모 두 輕症 및 中等症에 비해서 유의한 감소를 보였는데 반 하여 輕症과 中等症사이에는 $\mathrm{FEV}_{1} / \mathrm{FVC} \%, \dot{\mathrm{V}} \max 50 /$ $\mathrm{FVC} \%$, 및 $\dot{\mathrm{V}} \max 25 / \mathrm{FVC} \%$ 의 감소만이 유의하였다. 한편 $\mathrm{CV}$ 및 $\mathrm{CV} / \mathrm{VC}$ 는 병변의 범위에 따라 증가하는 경 향이 있었는데 重症에서의 $\mathrm{CV} / \mathrm{VC}$ 의 증가는 中等症 및 輕症에 비해서 유의하였다. 이러한 소견은 肺結核에서는 
병변의 범위가 크면 클 수록 심한 制限性換氣障碍와 더 불어 閉塞性換氣障碍도 동반되며 中等症에서는 輕症에 비해서 주로 細小氣道의 閉塞이 뚜렷하고, 重症에서는 輕 症 및 中等症에 비해 大氣道 및 細小氣道의 양자의 閉塞 이 현저함을 시사한다.

\section{REFERENCES}

1) Martin CJ, Pardee N, Dominik J: The diffuse obstructive pulmonary syndrome, I. Natural history. Am Rev Resp Dis 93:383, 1966

2) Pardee N, Dominik J, Martin CJ: The diffuse obstructive pulmonary syndrome, II. Factors associated with its progress. Am Rev Resp Dis 93:390, 1966

3) Martin CJ, Cochran TH, Katsura S: Tuberculosis, emphysema, and bronchitis. Am Rev Resp Dis 97: 1089, 1968

4) Simpson DG, Kuschner M, McClement J: Respiratory function in pulmonary tuberculosis. Am Rev Resp Dis 87:1, 1963

5) Snider GL, Doctor L, Demes TA, Shaw AR: Obstructive airway disease in patients with treated pulmonary tuberculosis. Am Rev Resp Dis 103:625, 1971

6) Krishna K, Bond S, Artvinli M, Reid KDG, McHardy GJR, Crofton JW: Pulmonary function in treated tuberculosis. Am Rev Resp Dis 115:40, 1977

7) Ahn CH, Nash DR, Hurst GA: Ventilatory defects in atypical mycobacteriosis, a comparison study with tuberculosis. Am Rev Resp Dis 113:273, 1976

8) Hallet WY, Martin CJ: The diffuse obstructive pulmonary syndrome in a tuberculosis sanatorium, I . Etiologic factors. Ann Intern Med 54:1146, 1961

9) Martin CJ, Hallet WY: The diffuse obstructive pulmonary syndrome in a tuberculosis sanatorium, II. Incidence and symptoms. Ann Intern Med 54: 1156, 1961

10) Martin CJ, Hallet WY: The diffuse obstructive pulmonary syndrome, a factor in tuberculosis treatment failures. Am Rev Resp Dis 86:898, 1962

11) Anno H, Tomashefski JF: Studies on the impairment of respiratory function in pulmonary tuberculosis. Am Rev Tuberc 71:333, 1955

12) Lancaster JF, Tomashefsky JF: Tuberculosis-a cause of emphysema. Am Rev Resp Dis 87:435, 1963

13) Malik SK, Martin CJ: Tuberculosis, corticosteroid therapy, and pulmonary function. Am Rev Resp Dis 100:13, 1969

14) Korol E: Pulmonary emphysema in tuberculosis. Am Rev Resp Dis 38:594, 1938

15) Katz J, Kunofsky S: Tuberculosis as a cause of the increasing mortality from emphysema. Am Rev Resp Dis 89:673, 1964

16) 朴熙明：結核性肺肋膜疾患의 肺臟機能에 관한 研究. 大邱醫學雜誌 $3: 1,1961$

17) 金萬在, 朴熙明：肺結核患者의 肺容量 吕 肺內 GAS 混合에 관한 研究, 第 I 編 病變의 輕重과 肺容量 및 그 區分. 大韓內科學會雜誌 5:665, 1962

18）金萬在, 朴熙明：肺結核患者의 肺容量 哭 肺內 GAS 混合에 관한 研究, 第II編. 換氣障碍의 病型과 肺容量 및 工 區分. 天韓內科學會雜誌 5:731, 1962

19) 金萬在, 朴熙明：肺結核患者의 肺容量 哭 肺內 GAS 混合에 관한 研究, 第III編, 殘氣率 品 換氣障碍의 病 型斗 肺內 GAS 混合. 大韓內科學會雜誌 5:797, 1962

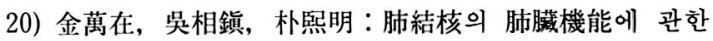
研究, 특히 換氣障碍의 病型을 中心으로. 結核 $12: 38$, 1962

21) Jayamanne DS, Epatein H, Goldring RM: The influence of lung volume on expiratory flow rates in diffuse interstitial lung disease. Am J Med Scien 275:329, 1978

22) Diagnostic standards and classification of tuberculosis. National tuberculosis and respiratory disease association, p 68, New York, 1961

23) 趙東奎, 朴熙明 : 換氣力學檢査의 推定正常値卅 관한 㖃究, I. 努力性呼氣曲線의 分析을 中心으로. 大韓醫 學協會誌 23:715, 1980

24) Anthonisen NR, Danson J, Robertson PC, Ross WRD: Airway closure as a function of age. Resp Physiol 8:58, 1979

25）李重基, 丁泰勳, 朴熙明：非吸煙 男女의 closing volume의 推定正常值에 관한 研究. 大韓內科學會雜誌 24:653, 1981

26) 李炳洙, 金重玖, 權寧輳, 丁泰勳, 李長白, 朴熙明：非 吸煙成人肺活量의 推定正常值. 大韓內科學會雜誌 23 : 143,1980

27）丁泰動, 全在殷, 李長白, 朴熙明：換氣力學檢査의 推 定正常值에 관한 研究, II. 最大呼氣流量曲線의 分析 을 中心으로 大韓醫學協會誌 23:985, 1980

28）鄭萬洪, 丁泰勳, 朴熙明: 男女吸煙者의 換氣力學檢査 成績의 推定正常值. 大韓醫學協會誌 29:515, 1986

29) Bell JW: Experimental pulmonary emphysema, production of emphysematous bullae in the rabbit by infection with tuberculosis. Am Rev Tuberc 78: 
848, 1958

30) Goensler EA, Lindgren I : Chronic bronchitis as an etiologic factor in obstructive emphsema. Am Rev Resp Dis 80:185, 1959

31) Curtis JK, Rasmussen HK, Medenhall JT: Detection of early pulmonary emphysema. Am Rev Tuberc 72: 569, 1955

32) Curtis JK, Rasmussen HK, Bauer H, Cree E: Recognition of early emphysema by pulmonary function tests. Chest 38:285, 1960

33) Hutchinson J: On the capacity of the lungs, and on the respiratory frnctions, with a view to establishing a precise and easy method of detecting desease by the spirometer. Tr Med Chir Soc London 29:137, 1846

34) Goldsmith JR: A simple test of maximal expiratory flow for detecting ventilatory obstruction. Am Rev Tuberc 78:180, 1958

35) Williams MH, Seriff NS, Akyol T, Yoo OH: The diffusing capacity of the lung in acute pulmonary tuberculosis. Am Rev Resp Dis 84:814, 1961

36) Marcus $\mathrm{H}$, Yoo $\mathrm{OH}$, Akyol T, Willians $\mathrm{MH}$ : A randomized study of the effects of corticosteroid therapy on healing of pulmonary tuberculosis as judged by clinical, roentgenographic, and physiologic measurements. Am Rev Resp Dis 88:55, 1963

37) Takishima T, Sasaki T, Takahashi K, Sasaki H, Nakamura T: Direct writing recorder of the flow volume curve and its clinical application. Chest 61: 262,1972

38) Kryger M, Bode F, Antic R, Anthonisen N: Diagnosis of obstruction of the upper and central airways. Am J Med 61:85, 1976

39) Bass H: The flow volume loop, normal standards and abnormalities in chronic obstructive pulmonary disease. Chest 63:171, 1973

40) Clement J,Woestijne KP: Variability of maximum expiratory flow volume curves and effort independency. J Appl Physiol 31:55, 1971

41) McFadden ER, Lindon, DA: A reduction on maximum mid-expiratory flow rate. Am J Med 52:725, 1972

42) Woolcock AJ, Vincent NJ, Macklem PT: Frequency dependence of compliance as a test for obstruction in the small air ways. J Clin Invest 48 : 1097,1969

43) McCarthy DS, Spencer R, Greene R, Emili JM: Measurement of closing volume as a simple and sensitive test for early detection of small air way disease. Am J Med 52:747, 1972

44) Gelb AF, Molony PA, Klein E, Aronstam S: Sensitivity of volume of isoflow in the detection of mild air way obstruction. Am Rev Resp Dis 112:401, 1975

45) Dosman J, Bode F, Urbanetti J, Martin R, Macklem PT: The use of a helium-oxygen mixture during maximum expiratory flow to demonstrate obstruction on small airways in smokers. J Clin Invest 55: 1090, 1975

46) Macklem PT: Obstruction in small airways-a challenge to medicine. Am J Med 52:721, 1972

47) Curtis JK, Rasmussen HK: Significance of the terminal rise in the single-breath oxygen test. Am Rev Tuberc 75:745, 1957

48) Green M, Travis DM, Mead J: A simple measurement of phase IV (closing volume) using a critical orifice helium analyzer. J Appl Physiol 33:827, 1972

49) Zapletal A, Motoyama EK, Woestijne KP, Hunt VR, Bouhuys A: Maximum expiratory flow-volume curves and airway conductance in children and adolescents. J Appl Physiol 26:308, 1969

50）金福元, 盧龍鎬, 韓成九, 姜鸿模, 金建烈, 韓鉘徹 : 肺 換氣機能檢査에 대한 㸴究. Volume corrected $\mathrm{MEF}_{50}$ 의 診斷的 價值에 대하여. 大韓內科學會雜誌 24:1139, 1981 